\title{
Many Body Interactions and Lattice Dynamics of Gold
}

\author{
S.R.B.Thapa \\ Birendra Multiple Campus,Bharatpur,Chitwan,Nepal \\ Email: thapasrb@yahoo.com
}

\begin{abstract}
Lattice dynamics of gold is studied theoretically using phenomenological model. This model considers (i ion-ion and (ii) ionelectron interactions to evaluate the potential energy. Contribution to ion-ion interaction is the sum of two body central interactions between ions and many body interactions among ions. Contribution to potential energy due to ion-electron interactions is evaluated using screened coulomb potential. Using this model phonon frequencies of gold are computed at 48 non-equivalent points of Brillouin zones of crystal and compared with experimental points. Lattice specific heats at different temperatures are evaluated by dividing the frequency spectrum in interval of 0.1 tiga hertz. Using the computed values of lattice specific heats, Debye characteristic temperatures of gold are computed. The model is used to calculate compressibility and Poission's ratio. Agreement of theoretical calculations with experimental results is satisfactory.
\end{abstract}

Key words: Ion- ion interaction, Ion-electron interaction, Phonon frequency, Lattice specific heat, Debye characteristic temperature.

\section{Introduction}

Theoretical study of lattice dynamics of metals has been done by various workers by using phenomenological models and pseudo potential approaches. Closs and Shukla (1977), Kumar and Jokadi (1975), Machado and Shukla (1976), Rathore(1977),Bertolo and shukla(1975), Rai and Hemkar(1976), Kharoo et al (1977), Thakur and Singh (1986). Singh and Prakash (1975) studied lattice dynamics of gold using model potential or pseudo potential approaches. Such approaches involve model or pseudo potential and the dielectric screening function with or without exchange and correlations among conduction electrons. Numerical calculations of different properties of metals from this theory are long and tedious. Phenomenological models are comparatively simple and are still of interest for the study of lattice dynamics of metals. In this context, using axially symmetry model for ionion interactions and screened Coulomb potential for ion-electron interactions, lattice dynamics of f.c.c. metals $\mathrm{Al}$ and $\mathrm{Pb}$ are studied by Thapa et al (2000). A phenomenological model; which considers two body central interactions between ions, many body interactions among ions and screened Coulomb potential forion-electron interactions; is developed by Thapa and Singh(1998) to study the lattice dynamics of face centered cubic metals $\mathrm{Al}$ and $\mathrm{Pb}$. This model is extended by Thapa(1998) to explain the thermo physical properties of transition metals palladium, thorium and nickel. In this present paper that same model is used to compute phonon frequencies, lattice specific heats, Debye Characteristic temperatures, Compressibility and Poisson's ratio of gold.

\section{Theoretical Model}

The Secular determinant which determines the angular frequency ' $\omega$ ' of mode of vibration in the crystal is given by

$$
\mathrm{D}(\mathbf{q})-\omega \mathrm{I} \mid=0
$$

Where $\mathrm{m}$ is ionic mass and $\mathrm{I}$ is unit matrix and $\mathrm{D}(\mathbf{q})$ is $3 \times 3$ dynamical matrix. The elements of dynamical matrix $\mathrm{D}(\mathbf{q})$ are written as

$$
\mathrm{D}(\mathbf{q})=\mathrm{D}(\mathbf{q})^{\mathrm{i}-\mathrm{i}}+\mathrm{D}(\mathbf{q})^{\mathrm{i}-\mathrm{e}}
$$

Superscripts $\mathrm{i}-\mathrm{i}$ and $\mathrm{i}$-e represent ion- ion and ionelectron interactions respectively.

$$
\mathrm{D}(\mathbf{q})^{\mathrm{i}-\mathrm{i}}=\left[\mathrm{D}(\mathbf{q})^{\mathrm{i}-\mathrm{i}}\right]^{\mathrm{c}}+\left[\mathrm{D}(\mathbf{q})^{\mathrm{i}-\mathrm{i}}\right]^{\mathrm{m}}
$$

Superscripts $\mathrm{c}$ and $\mathrm{m}$ represent two body central interactions between ions and many body interactions among ions respectively. To evaluate the secular determinant central forces between nearest and next nearest neighbours of the reference atom are taken into account.

Dynamical matrix for two body central interaction of ions is given by

$$
\begin{aligned}
& {\left[\mathrm{D}_{\mathrm{ii}}(\mathbf{q})^{\mathrm{i}-\mathrm{i}}\right]^{\mathrm{c}}=1 / \mathrm{m}\left[4 \alpha_{1}-2 \alpha_{1} \mathrm{C}_{\mathrm{i}}\left(\mathrm{C}_{\mathrm{j}}+\mathrm{C}_{\mathrm{k}}\right)+\alpha_{1} \alpha_{2}\right]---(4)} \\
& {\left[\mathrm{D}_{\mathrm{ij}}(\mathbf{q})^{\mathrm{i}-\mathrm{i}}\right]^{\mathrm{c}}=\left(2 \alpha_{1} / \mathrm{m}\right) \mathrm{S}_{\mathrm{i}} \mathrm{S}_{\mathrm{j}} \quad(\mathrm{i} \neq \mathrm{j})}
\end{aligned}
$$

Superscripts $i, j$ and $k$ are indices. $\alpha_{1}$ and $\alpha_{2}$ are force constants.

$$
\mathrm{C}_{1}=\operatorname{Cos}\left\{\mathrm{q}_{1}(\mathrm{a} / 2)\right\}, \mathrm{S}_{1}=\operatorname{Sin}\left\{\mathrm{q}_{1}(\mathrm{a} / 2)\right\} \quad(\mathrm{l}=\mathrm{i}, \mathrm{j}, \mathrm{k})
$$

Dynamical matrix for many body interactions among ions is given by Sarkar and Sengupta 1969, 
$\left[D_{\text {ii }}(\mathbf{q})^{\mathrm{i} i \mathrm{i}}\right]^{\mathrm{m}}=1 / \mathrm{m}\left[8 \mathrm{P} \operatorname{Sin}^{2}\left\{\mathrm{q}_{\mathrm{i}}(\mathrm{a} / 2)\right\}-4 \mathrm{P}\left[\operatorname{Sin}^{2}\left\{\mathrm{q}_{\mathrm{i}}(\mathrm{a} / 2)\right\}+\operatorname{Sin}^{2}\left\{\mathrm{q}_{\mathrm{k}}(\mathrm{a} / 2)\right\}\right]\right.$

$-2 \mathrm{P} \operatorname{Cos}\left(\mathrm{q}_{\mathrm{i}} \mathrm{a}\right)\left[\operatorname{Cos}\left(\mathrm{q}_{\mathrm{j}} \mathrm{a}\right)+\operatorname{Cos}\left(\mathrm{q}_{\mathrm{k}} \mathrm{a}\right)\right]+8 \mathrm{P} \operatorname{Cos}\left(\mathrm{q}_{\mathrm{i}} \mathrm{a} / 2\right) \operatorname{Cos}\left(\mathrm{q}_{\mathrm{j}} \mathrm{a}\right) \operatorname{Cos}\left(\mathrm{q}_{\mathrm{k}} \mathrm{a}\right)$ $(40 \mathrm{P}-22 \sqrt{2} \mathrm{Q}) \operatorname{Cos}\left\{\mathrm{q}_{\mathrm{i}}(\mathrm{a} / 2)\right\}\left[\operatorname{Cos}\left\{\mathrm{q}_{\mathrm{j}}(\mathrm{a} / 2)\right\}+\operatorname{Cos}\left\{\mathrm{q}_{\mathrm{k}}(\mathrm{a} / 2)\right\}\right]+44 \sqrt{2} \mathrm{Q}$ $\left.\operatorname{Cos}\left\{\mathrm{q}_{\mathrm{j}}(\mathrm{a} / 2)\right\} \operatorname{Cos}\left\{\mathrm{q}_{\mathrm{k}}(\mathrm{a} / 2)\right\}-84 \mathrm{P}+88 \sqrt{2} \mathrm{Q}\right]$

Here $\mathrm{P}$ and $\mathrm{Q}$ are force constants.

$\left[D_{i j}\left(q^{i-i}\right]^{m}=1 / m\left[2 P \operatorname{Sin}\left(q_{i} a\right) \operatorname{Sin}\left(q_{i j} a\right)+4 P \operatorname{Sin}\left\{q_{i}(a / 2)\right\} \operatorname{Sin}\left\{q_{j}(a / 2)\right\}\right.\right.$ $+4 P \operatorname{Cos}\left\{q_{k}(a / 2)\right\}\left[\operatorname{Sin}\left(q_{i} a\right) \operatorname{Sin} q_{j}(a / 2)+\operatorname{Sin}\left(q_{j} a\right) \operatorname{Sin}\left\{q_{i}(a / 2)\right]\right.$ $\left.+4 P \operatorname{Sin}\left\{q_{i}(a / 2)\right\} \operatorname{Sin}\left\{q_{k}(a / 2)\right\}+(40 P+22 \sqrt{ } 2 Q) \operatorname{Sin}\left\{q_{i}(a / 2)\right\} \operatorname{Sin}\left\{q_{j}(a / 2)\right\}\right]--(6)$

Other elements of dynamical matrix for the many body interaction can be written by the cyclic permutation of indices $i, j$ and $\mathrm{k}$. Matrix elements corresponding to ion-electron interaction considering screened coulomb potential on the basis of Kreb's approach (1964) is given by Thapa et al (2003) as:

$\left[D_{11}\right]^{\mathrm{i}-\mathrm{e}}=\mathrm{A}\left[\sum_{\mathbf{h}} \frac{\left(\mathbf{q}_{1}+\mathbf{h}_{1}\right)^{2} G^{2}\left(x_{1}\right)}{|\mathbf{q}+\mathbf{h}|^{2}+K_{e}^{2} f\left(t_{1}\right)}-\sum_{\mathbf{h} \neq 0} \frac{\mathbf{h}_{1}^{2} G\left(x_{2}\right)}{\mid \mathbf{h}^{2}+K_{e}^{2} f\left(t_{2}\right)}\right]$

$\left[\mathrm{D}_{11}\right]^{\mathrm{ie}}$ represents diagonal elements of dynamical matrix $\mathrm{D}$ for ion-electron interaction.

$\left[D_{12}\right]^{i-e}=\mathrm{A}\left[\sum_{\mathbf{h}} \frac{\left(\mathbf{q}_{1}+\mathbf{h}_{1}\right)^{2}\left(\mathbf{q}_{2}+\mathbf{h}_{2}\right)}{|\mathbf{q}+\mathbf{h}|^{2}+K_{e}^{2} f\left(t_{1}\right)}-\sum_{\mathbf{h} \neq 0} \frac{\mathbf{h}_{2} G^{2}\left(x_{2}\right)}{\mid \mathbf{h}^{2}+K_{e}^{2} f\left(t_{2}\right)}\right]$

$\left[\mathrm{D}_{12}\right]^{\text {i-e }}$ represents off diagonal elements of dynamical matrix D for ion-electron interaction. Other matrix elements may be obtained by cyclic permutations of indices. Summation extends over reciprocal lattice vector $\mathbf{h}$.

$G(x)=3\left[\frac{\operatorname{Sin} x-x \operatorname{Cos} x}{x^{3}}\right] x_{1}=|\mathbf{q}+\mathbf{h}| r_{e} \quad x_{2}=|\mathbf{h}| r_{e} \quad f(t)=0.5+\left[\frac{1-t^{2}}{4 t}\right] \mathrm{h}\left[\frac{1+t}{1-t}\right]$ $\mathrm{t}_{1}=\frac{|\mathbf{q}+h|}{2 \mathbf{k}_{F}}, t_{2}=\frac{|\mathbf{h}|}{2 \mathbf{k}_{F}} \mathrm{r}_{\mathrm{e}}$ is inter electronic spacing defined as $r_{e}=\left[\frac{3}{4 \pi n_{e}}\right.$ from Thakur and Singh(1986). $\mathrm{K}_{\mathrm{F}}$ is Fermi surface wave vector.

$n_{e}$ is electron density. For monovalent metals $r_{e}$ is equal to radius of atomic sphere $r_{0}$. For polyvalent metals $\mathrm{r}_{\mathrm{e}}=Z^{\frac{-1}{3}} r_{o}$ with $\mathrm{Z}$ as valency. $\mathrm{K}_{\mathrm{e}}$ is screening parameter given by .

$$
K_{e}=\sigma \frac{r_{e}}{a_{o}} K_{F}
$$

$\mathrm{a}_{\mathrm{o}}$ is Bohr radius. $\mathrm{K}_{\mathrm{e}}$ has been treated as adjustable parameter. Value of $\sigma$ is adjusted to give best fit with experimental data. A is structure dependent constant.

\section{Numerical Computations}

The secular determinant $\mid \mathrm{D}(\mathbf{q})$ - $\omega \mathrm{I} \mid=0$, is solved along symmetry direction $[\xi 00]$. It is also solved for long wavelength $\operatorname{limit}(\mathbf{q} \rightarrow 0)$ to obtain expressions for elastic constants in terms of force constants $\left(\mathrm{P}, \mathrm{Q}, \alpha_{1}\right.$ and $\left.\alpha_{2}\right)$ and bulk modulus of electron gas $\mathrm{K}_{\mathrm{e}}$, which are given below:

$\mathrm{C}_{11}=1 / \mathrm{a}\left[2 \alpha_{1}+4 \alpha_{2}+72 \mathrm{P}-22 \sqrt{2} \mathrm{Q}\right]+\mathrm{K}_{\mathrm{e}}$
$\mathrm{C}_{12}=1 / \mathrm{a}\left[\alpha_{1}+36 \mathrm{P}-55 \sqrt{2} \mathrm{Q}\right]$
$\mathrm{C}_{44}=1 / \mathrm{a}\left[\alpha_{1}+32 \mathrm{P}-33 \sqrt{2} \mathrm{Q}\right]$

The frequencies of the normal modes of vibration at the zone boundary along the $[\xi 00]$ direction are given by, $m \omega_{\mathrm{L}}^{2}(\mathrm{x})=8 \alpha_{1}-8 \mathrm{P}+88 \sqrt{ } 2 \mathrm{Q}+\mathrm{D}_{11}^{\mathrm{i}-\mathrm{e}}$

Subscript L represents longitudinal wave, is angular $^{2}$ frequency. $\mathrm{D}_{11}{ }^{\mathrm{i}-\mathrm{e}}$ is dynamical matrix corresponding to ion-electron interaction. Model parameters are evaluate by using following data in equations (1),(2),(3) and (4). $\mathrm{D}_{11}{ }^{\mathrm{ie}}$ is evaluated by technique of Thakur and Singh (1986).

Table-1(Elastics constants of Gold)

\begin{tabular}{|l|l|c|c|l|}
\hline Metal & \multicolumn{3}{|l|}{ Elastic constants $\left(10^{10} \mathrm{Nm}^{-2}\right)$} & References \\
& $\mathrm{C}_{11}$ & $\mathrm{C}_{12}$ & $\mathrm{C}_{44}$ & Neighbours and \\
Gold & 19.234 & 16.314 & 4.195 & \begin{tabular}{l} 
Alers(1958) \\
\hline
\end{tabular} \\
\hline
\end{tabular}

Table-2(zone boundary phonon frequencies of Gold)

\begin{tabular}{|c|c|c|c|}
\hline Metal & $\begin{array}{l}\text { Zone } \\
\text { Frequ } \\
\left.v_{L}\right)_{X}\end{array}$ & $\begin{array}{l}\text { ry phonon } \\
\left(0^{12} \mathrm{~Hz}\right) \\
\left.. \mathrm{U}_{\mathrm{T}}\right)_{\mathrm{X}}\end{array}$ & References \\
\hline Gold & 4.61 & 2.75 & 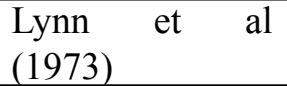 \\
\hline
\end{tabular}

Table-3 (Atomic mass, Semi-lattice constant, screening multiplication constant, Interelectronic spacing, Fermi surface wave vector)

\begin{tabular}{|c|c|c|c|c|c|c|}
\hline 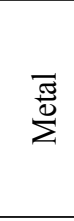 & 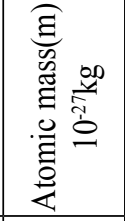 & 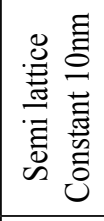 & 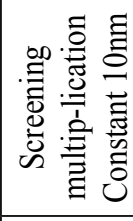 & 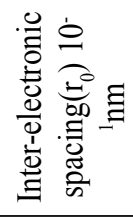 & 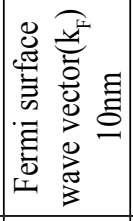 & 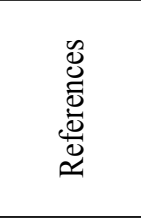 \\
\hline Gold & 316.965 & 2.035 & 0.353 & 1.592 & 1.204 & $\begin{array}{l}\text { Lynn et } \\
\operatorname{al}(1973)\end{array}$ \\
\hline
\end{tabular}

Evaluated values of model parameters are given below: 
Thapa/Many Body...

\begin{tabular}{|c|c|c|c|c|c|}
\hline \multicolumn{6}{|c|}{ Table-4(Evaluated values of model parameters) } \\
\hline \multirow{2}{*}{ Metal } & \multicolumn{4}{|c|}{ Force constants $\left(\mathrm{Nm}^{-1}\right)$} & \multirow{2}{*}{$\begin{array}{l}\text { Bulk } \\
\text { M od ulus } \\
\mathrm{K}_{\mathrm{e}} \\
\left(10^{11} \mathrm{Nm}^{-2}\right)\end{array}$} \\
\hline & $\alpha_{1}$ & $\alpha_{2}$ & $\mathrm{P}$ & Q & \\
\hline Gold & 30.8610 & -4.7620 & 0.22853 & -0.09830 & 5.234 \\
\hline
\end{tabular}

Using the evaluated values of force constants phonon dispersion relations of gold along symmetry directions $[\xi 00],[\xi \xi 0]$ and $[\xi \xi \xi]$ are computed.

The values of $\mathrm{C}_{\mathrm{v}}$ at different temperatures are obtained by formula from Thapa and Singh (1998)

$$
\mathrm{C}_{\mathrm{v}}=(3 \mathrm{R} / 3000) \sum \mathrm{E}(\mathrm{hu} / \mathrm{kT}) \mathrm{g}(\mathrm{v})
$$

$\mathrm{g}(\mathrm{v})$ is frequency distribution function defined by $g(v) d v=9 N v^{2} d v / v_{D}{ }^{3}, v_{D}$ is Debye threshold frequency, $v_{D}=k \theta_{D} / h$. $E(h v / k T)$ is the Einstein function defined by $E_{x}=\left(x^{2} e^{x}\right) /\left(e^{x}-1\right)^{2}$ where $x=h v /$ $\mathrm{kT}, \mathrm{v}$ is frequency, $\mathrm{k}$ is Boltzmann constant, $\mathrm{h}$ is Planck's constant, $\mathrm{T}$ is temperature and $\mathrm{N}$ is number of atoms in the crystal. $\theta_{D}$ is Debye temperature. Calculation of $\mathrm{C}_{\mathrm{V}}$ at different temperatures is done by dividing first Brillouin zone of the crystal into one thousand miniature cells which reduce to 48 non equivalent points under symmetry operations. Secular equation is solved at these points by dividing the frequency spectrum in intervals of $\Delta v=0.1 \mathrm{THz}$. Debye temperature $\theta_{\mathrm{D}}$ is calculated by standard table of $\left[\mathrm{C}_{\mathrm{V}}-\theta_{\mathrm{D}} / \mathrm{T}\right]$ from Saha and Srivastava (1965) by using computed values of $\mathrm{C}_{\mathrm{v}}$ at different temperatures. Compressibility is calculated by using formula $\mathrm{K}=3 /\left(\mathrm{C}_{11}+2 \mathrm{C}_{12}\right)$ while Poisson's ratio is calculated by formula $\sigma=\mathrm{C}_{12} /\left(\mathrm{C}_{11}+\mathrm{C}_{12}\right)$

Tabel-5 (Calculated and Observed values of compressibility in units of $\left(10^{-11} \mathrm{~N}^{-1} \mathrm{~m}^{2}\right)$

\begin{tabular}{|c|c|c|c|}
\hline Metal & Calculated value & Observed value & Reference \\
\hline Gold & 0.472 & 0.577 & Kittel(1976) \\
\hline
\end{tabular}

Tabel-6(Poisson's ratio)

\begin{tabular}{|c|c|c|c|}
\hline Metal & $\begin{array}{c}\text { Calculated } \\
\text { value (this } \\
\text { work) }\end{array}$ & $\begin{array}{c}\text { Calculated } \\
\text { value (from } \\
\text { experimental data) }\end{array}$ & Reference \\
\hline Gold & 0.5353 & 0.4589 & $\begin{array}{c}\text { Neighbours and } \\
\text { Alers(1958) }\end{array}$ \\
\hline
\end{tabular}

\section{Results and Discussion:}

\section{Phonon frequencies}

Kumar and Hemkar (1978) used short range paired forces in conjunction with Kreb's , model for ion electron interaction in the study of phonon dispersion relations of gold.

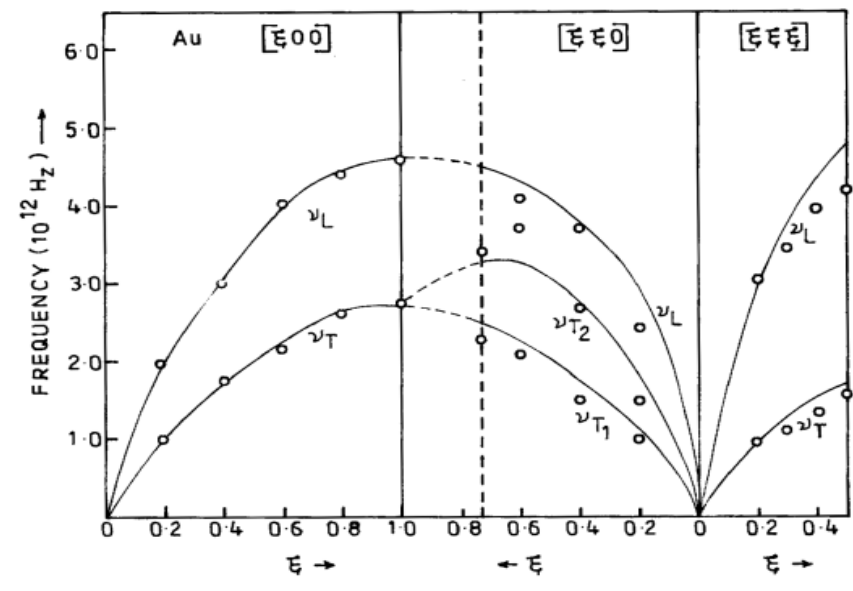

Fig.1 Phonondispersioncurveforgoldalongsymmetry directions Expt point are due to Lynn et all 1973.

Lynn et al (1973) measured the phonon frequencies of gold along principal symmetry directions at room temperature by the coherent inelastic scattering of neutron. . Nand and Tripathi (1975) studied lattice dynamics of gold to study phonon dispersion curves using pseudo potential approach. Thakur and Singh (1986) computed phonon frequencies using valence force field model. Phonon dispersion curves of gold have been computed assuming central and angular volume interactions by Rothore(1977). Calculated phonon frequencies according to the present model are plotted against reduced wave vector in figure (1) along with experimental points of Lynn et al (1973). Satisfactory agreement is found. Present result also compares well with those of other reported works of Kumar and Hemkar(1978) and Thakur and Singh (1986).

\section{Lattice specific heat}

Many theoretical workers calculated specific heats of gold. On the basis of non-central force model, Machado and Shukla(1976) also evaluated lattice specific heat of gold. Kharoo et al (1977) exploited the improved CGW model with Kreb's type volume force to calculate specific heat of gold. Thakur and Singh 
(1986) reported results of specific heat capacity of gold accurately within the range of $15-300 \mathrm{k}$ and results obtained satisfied the earlier experimental results of Culsius and Hartek (1928). Martin(1987) measured specific heat of gold below 300K. By applying Kreb's model(1964), Shukla (1965) evaluated lattice specific heat capacity of gold.

Calculated specific heats of gold(on the basis of adopted model)as a function of temperature are plotted in figure(2) with experimental points of Geballe and Giaque (1952) giving satisfactory agreement. Present results also compare well with other calculated results of Thakur and Singh(1986).

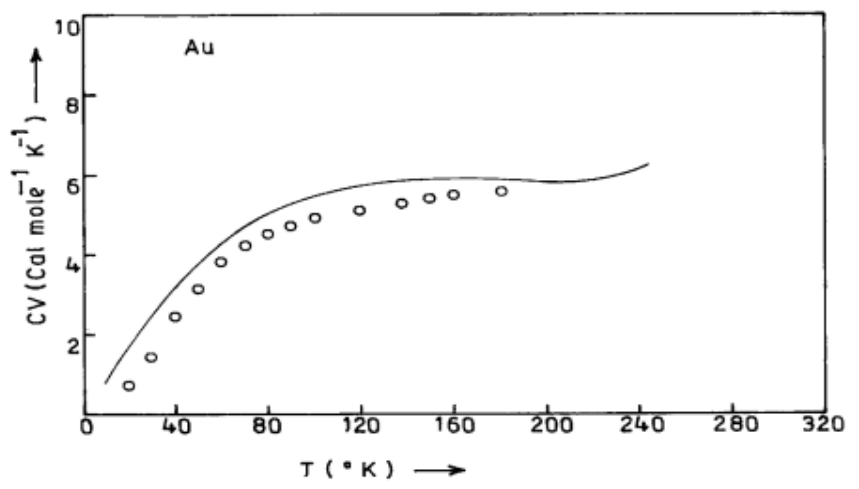

Fig.2 Specific heat capacity versus temperature curve for gold. Experimental points (0) are due to Geballe and Giaque (1952).

\section{Debye Characteristic temperatures}

Calculated Debye Characteristic temperatures of gold along with experimental points of Geballe and Giaque(1952) are plotted against temperature in fig (3).Agreement between calculated and experimental results is satisfactory. Present results compare well with results of Thakur and Singh (1986).

\section{Compressibility and poisson's ratio}

Calculated results of compressibility and poisson's ratio are in satisfactory agreement with experimental results.

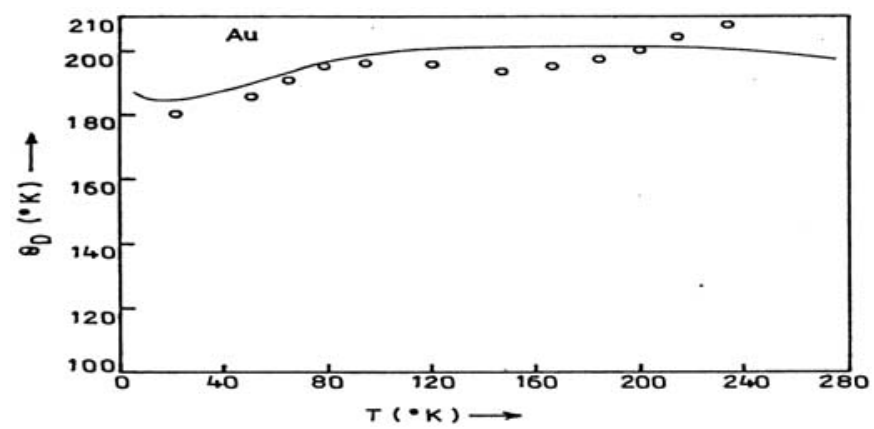

Fig.3. Debye temp versus temp curve for gold. Expt points (0) are due to Geballe and Giaque 1952.

\section{Conclusions}

The present theoretical model used to study the lattice dynamics of $\mathrm{Al}$ and $\mathrm{Pb}$ and transition metals explains satisfactorily phonon dispersion results, Lattice specific heats, Debye characteristic temperature, Compressibility and Poisson's ratio of noble metal gold. Thus two body and many-body interaction for ions and screened Coulomb potential for ion-electron interaction is successful in explaining the lattice dynamics of noble metal gold. (Elaborate more)

\section{References}

Bertolo, L. A.and Shukla, M. M. 1975. Acta Physical Academic Sc.Hung.39:49.

Closs,H. and Shukla, M.M. 1977.Nuovo Cimento 42B:213.

Clusius, K. and P Hartek. 1928.Z.Physik Chem.134:243.

Geballe, J. H. and Giaque, W. F. 1952.J.Amer.Chem. Soc.71:2368.

Kharoo,H.L., Gupta,O.P. and Hemkar, M.P. 1977.Helv.Phys. Acta Switzerland 50:545.

Kittel,C.1976. Introduction to Solid state physics.Wiley Eastern Limited $5^{\text {th }}$ edition .pp. 31 .

Krebs, K.1964. Physics Letters 10:12.

Kumar, M. and Hemkar, M.P. 1978.Nuovo Cimento 44B:451.

Kumar,S. and Jokadia, S. 1975.Solid State Commun.16:255.

Lynn,J.W, Smith, H.G. and Nicklow,R.M. 1973.Phy.Rev. B8:3493.

Machado,J.F. and Shukla, M.M. 1976.Acta Physical Academic Sc.Hung.40:43.

Martin, Douglas L.1987. Canad.J. Phys65:1104.

Nand S. and Tripathy B.B.1975.Physics letters 53A:229.

Neighbours,J.F. and G.A.Alers 1958.Phys.Rev.111:707.

Rai,R.C.and M.P.Hemkar 1976.Ind.J.Pure\& Appl. Phys.14:976.

Rathore, R. P.S.1975.Indian J. Phys. 49:858.

Rathore,R.P.S.1977.Ind.J.Pure\& Appl.Phys 15:54.

Saha, M.N.and B.N.Srivastava 1965.A Treatise on Heat,pp $713,5^{\text {th }}$ ed .Indian Press,Allahabad.

Sarakar, A.K.and Sengupta, S. 1969. Phy.Stat.Sol.36:359

Shukla, M. M.1965. Phys. Stat. Sol.8:475.

Shukla,N.and Prakash, S. 1975.Phys.Stat.Sol.8:475.

Singh.N.and Prakash, S. 1975.Phys.Rev.B12:1600.

Thakur,V.K.\& Singh,T.N.1986.Phys.Stat.Sol(b)135:67.Thapa, S.R.B.1998.journal of NPS 15:22.

Thapa,S.R.B.and Singh,T.N. 1998.Journal of NPS 15:45.

Thapa,S.R.B, Singh, O.P. and Singh, T.N. 2000.Nepal Journal of Sc. and Tech.2:31. 